\title{
Prostatic artery embolization: magnetic resonance image (MRI) findings in the early detection of prostate infarction in a canine spontaneous benign prostatic hyperplasia model
}

\author{
Vanesa Lucas-Cava ${ }^{1} \wedge$, Francisco Miguel Sánchez-Margallo ${ }^{2} \wedge$, Virginio García-Martínez $^{3}$, \\ Carmen López-Sánchez ${ }^{3}$, Claudia Báez-Díaz ${ }^{1}$, Luis Dávila-Gómez ${ }^{4} \wedge$, Juan Rafael Lima-Rodríguez ${ }^{5}$, \\ Fei $\operatorname{Sun}^{1 \wedge}$
}

${ }^{1}$ Endoluminal Therapy and Diagnosis Unit, Jesús Usón Minimally Invasive Surgery Centre, Cáceres, Spain; ${ }^{2}$ Scientific Director, Jesús Usón Minimally Invasive Surgery Centre, Cáceres, Spain; ${ }^{3}$ Human Anatomy and Embryology, Faculty of Medicine, University of Extremadura, Badajoz, Spain; ${ }^{4}$ Animal Housing Service, Jesús Usón Minimally Invasive Surgery Centre, Cáceres, Spain; ${ }^{5}$ Anaesthesiology Unit, Jesús Usón Minimally Invasive Surgery Centre, Cáceres, Spain

Contributions: (I) Conception and design: F Sun, V Lucas-Cava; (II) Administrative support: F Sun, V Lucas-Cava; (III) Provision of study materials or patients: All authors; (IV) Collection and assembly of data: F Sun, V Lucas-Cava; (V) Data analysis and interpretation: F Sun, V Lucas-Cava; (VI) Manuscript writing: All authors; (VII) Final approval of manuscript: All authors.

Correspondence to: Vanesa Lucas-Cava. Endoluminal Therapy and Diagnosis Unit, Jesús Usón Minimally Invasive Surgery Centre, Road N-521, km 41.8, 10071 Cáceres, Spain. Email: vlucas@ccmijesususon.com; Francisco Miguel Sánchez-Margallo. Scientific Director, Jesús Usón Minimally Invasive Surgery Centre, Road N-521, km 41.8, 10071 Cáceres, Spain. Email: msanchez@ccmijesususon.com.

Background: The purpose was to assess the association between prostate infarction and prostate volume $(\mathrm{PV})$ reduction after prostatic artery embolization (PAE) and define the best time point in detection of prostate infarction.

Methods: Ten male beagles (3.5-6.4 years) with spontaneous benign prostatic hyperplasia (BPH) underwent PAE. Magnetic resonance image (MRI) was conducted immediately before and 1 week, 2 weeks and 1 month after PAE to document prostate infarcts and measure PV. The sum of infarct areas (SUMIA) was measured and calculated using OsiriX software. Spearman's rank correlation was used to estimate the relationship of PV reduction rate with infarction percentage and SUMIA reduction.

Results: In comparison with baseline data, significant PV reduction $(\mathrm{P}<0.001)$ occurred at 2 weeks and continued to decrease substantially $(\mathrm{P}=0.004)$ from 2 weeks to 1 month after PAE. In the same fashion, significant decrease in both SUMIA and infarction percentage was observed from 1 to 2 weeks $(\mathrm{P}=0.002)$, and subsequently to 1 month ( $\mathrm{P}=0.039$ and $\mathrm{P}=0.016$, respectively). Spearman's rank correlation test demonstrated infarction percentage at 1 week had a stronger correlation $(\mathrm{r}=0.880, \mathrm{P}=0.001)$ with $\mathrm{PV}$ reduction rate at 1 month than infarction percentage at 2 weeks $(\mathrm{r}=0.733, \mathrm{P}=0.016)$. PV reduction rate had a significant correlation with decrease in SUMIA ( $\mathrm{r}=0.854, \mathrm{P}=0.002)$ at 1 month after PAE.

Conclusions: One week after PAE is an ideal time point to evaluate prostate infarction. Prostate infarction percentage at 1 week is a good predictor for prostate shrinkage at 1 month after PAE.

Keywords: Benign prostatic hyperplasia (BPH); canine model; magnetic resonance image (MRI); prostate infarction; prostatic artery embolization (PAE)

Submitted Oct 07, 2020. Accepted for publication Dec 22, 2020.

doi: $10.21037 /$ tau-20-1320

View this article at: http://dx.doi.org/10.21037/tau-20-1320

\footnotetext{
^ ORCID: Vanesa Lucas-Cava, 0000-0002-9807-386X; Francisco Miguel Sánchez-Margallo, 0000-0003-2138-988X; Virginio GarcíaMartínez, 0000-0002-7760-1985; Carmen López-Sánchez, 0000-0003-3296-2071; Claudia Báez-Díaz, 0000-0001-9031-2929; Luis DávilaGómez, 0000-0002-6620-7050; Juan R. Lima-Rodríguez, 0000-0003-3710-6936; Fei Sun, 0000-0002-3865-3811.
} 


\section{Introduction}

Prostatic artery embolization (PAE) has been performed worldwide in the management of symptomatic benign prostatic hyperplasia (BPH) during the last decade. With increasing experience in clinical practice, investigation of predictive factors has gained more interest aiming to optimize the selection in patients who may benefit from PAE or individualize treatment plan based on anticipated prediction in the prognosis after PAE. Prostate infarction induced with PAE is a potential predictor, which has been demonstrated to have a strong correlation with prostate volume (PV) reduction (1), thus relieving lower urinary tract symptoms (LUTS) and bladder outlet obstruction (BOO) $(2,3)$. After initial studies on prostate infarction at 1 month after PAE in successful prediction of subsequent clinical outcomes $(4,5)$, clinicians suggested further studies in evaluation of the potential predictive role of prostate ischemia at the first 24 hours or 1-2 weeks after PAE (4). However, since the infarction progressively decreases in size and tends to disappear after the first month following PAE $(6,7)$, several attempts failed to validate its predictive role of prostate infarction at 24 hours $(8,9)$; and the best time point to detect the prostate infarction after PAE in evaluation of its predictive capability remains unclear. To elucidate the prostate infarct involution after PAE and provide experimental evidence for the best time point in detection of prostate infarction, the present study was conducted in a canine spontaneous $\mathrm{BPH}$ model with purpose to observe the early magnetic resonance image (MRI) findings of pathological reactions to $\mathrm{PAE}$, evaluate the changes in prostate infarct size with time, and identify the association between prostate infarction and PV reduction after PAE.

We present the following article in accordance with the ARRIVE reporting checklist (available at http://dx.doi. org/10.21037/tau-20-1320).

\section{Methods}

\section{Animals}

Experiments were performed under a project license (No. 2018209010001587 and No. 2018209010008108) granted by Regional Ethic Committee of Junta de Extremadura, in compliance with Spanish national guidelines for the care and use of animals. Ten adult male Beagle dogs with a mean body weight of $16.9 \pm 2.7 \mathrm{~kg}$ (standard deviation) were enrolled in this study. The mean age of beagles was $4.9 \pm 0.8$ years with a range of 3.5-6.4 years. All dogs suffered from spontaneously occurring $\mathrm{BPH}$ with $\mathrm{PV}$ larger than $18 \mathrm{~mL}$ (mean PV 19.69 $\pm 2.75 \mathrm{~mL}$ ), which was indicated as a gravimetric criterion in diagnosis of spontaneous BPH in beagles whereas the normal prostate size is generally less than $12 \mathrm{~mL}(10)$.

\section{PAE procedure}

After fasting for 24 hours, the animals were anesthetized and maintained with inhalation anesthesia. In a sterile manner, the right femoral artery access was established percutaneously. Under fluoroscopy, a 4-Fr angiographic catheter (Simmons Sidewinder I, Terumo Medical, Somerset, NJ, USA) was placed into the main trunk of the left and right internal iliac artery or its anterior branch. A 2.4Fr microcatheter (Progreat, Terumo Medical, Somerset, NJ, USA) with a $0.016 "$ microwire (GT, Terumo Medical, Somerset, NJ, USA) was inserted into the prostatic artery. After superselective angiography in the prostatic artery, embolization was performed in five dogs with HydroPearl ${ }^{\circledR}$ $(400 \pm 75 \mu \mathrm{m}$, Terumo, Tustin, CA, USA), four dogs with Embosphere ${ }^{\circledR}(300-500 \mu \mathrm{m}$, Merit Medical EMEA, Amerikalaan, The Netherlands), and one dog with LifePearl ${ }^{\circledR}$ $(400 \pm 50 \mu \mathrm{m}$, Terumo, Tustin, CA, USA). Embolization was terminated immediately when complete stasis was obtained or reflux of the mixture of embolic agent and contrast medium toward the caudal vesical artery, middle rectal artery or internal pudendal artery was observed. At completion of the intervention, the animals were recovered from general anesthesia. Clinical observation after PAE was checked twice a day for 1 week and then once daily until 1 month for potential complications associated with PAE.

\section{MRI evaluation}

MRI examinations were performed in all dogs with a 1.5T system (Intera; Philips Medical Systems, Best, The Netherlands) immediately before PAE as baseline data and 1 week, 2 weeks and 1 month after PAE. Under general anesthesia, the animals were placed in a supine position with a SENSE-Flex-M coil (Philips Medical Systems, Best, The Netherlands) on the lower abdomen for image acquisition. Acquisition was done in axial, coronal and sagittal T2-weighted turbo spin-echo sequences with major parameters: repetition time $(\mathrm{TR})=5,509$ milliseconds, echo time $(\mathrm{TE})=125$ milliseconds; echo-train length $=17$; field of view $=150 \mathrm{~mm}$; slice thickness $=3 \mathrm{~mm}$, gap $=0.3 \mathrm{~mm}$; and matrix $=224 \times 224$. The axial T1-weighted turbo 
spin-echo sequences were performed before and after gadolinium enhancement. Contrast-enhanced T1-weighted imaging was obtained by intravenous administration of a bolus of $0.1 \mathrm{mmol} / \mathrm{kg}$ gadopentetate dimeglumine (Magnevist $^{\circledR}$; Schering AG, Berlin, Germany). Image analysis was performed by a radiologist with more than 15 years of experience in prostate imaging (the last author) and a junior investigator with 1-year experience (1st author). The PV was obtained using the standard ellipsoid formula: length $\times$ width $\times$ height $\times \pi / 6$. Ischemia/infarct areas were determined on contrast-enhanced T1-weighted axial images and defined as new regions that displayed no evidence of enhancement after gadolinium administration. Measurement of infarct areas was performed with OsiriX 64-bit software (Pixmeo, Bernex, Switzerland). In the present study, sum of infarct areas (SUMIA) was used as a surrogate parameter to describe the size of prostate infarction, which was defined as the sum of total prostate infarct areas in all sections of the prostate. Sum of prostate areas (SUMPA) in all sections was also calculated. Prostate infarction percentage was defined as the ratio of SUMIA/SUMPA $\times 100 \%$.

\section{Statistical analysis}

The statistical analysis was conducted with statistical software package SPSS version 24. The descriptive analysis was performed in all variables and expressed as means \pm standard deviation. The statistical differences at various time points were assessed by the paired sample $t$-test for the mean PV and Wilcoxon signed-rank for the mean SUMPA, the mean SUMIA and prostate infarction percentage. Spearman's rank correlation and simple linear regression models were used to estimate the relationship between PV reduction rate and prostate infarction. $\mathrm{P}$ value $<0.05$ was considered to indicate a statistically significant difference.

\section{Results}

PAE was performed successfully in the prostatic arteries of both sides in all dogs. Control angiography immediately after embolization showed complete occlusion of the prostatic arterial trunk and its major branches on both sides in all cases. No major complications due to nontarget embolization were observed during 1-month follow-up.

\section{MRI morphological findings in canine $B P H$ before $P A E$}

MRI examination in dogs with spontaneous $\mathrm{BPH}$ prior to
PAE revealed the enlarged prostates with diffuse hyperplasia throughout the gland tissue, rather than the hyperplastic nodules as like in humans (Figure 1A,B,C). The canine prostate has a symmetric two-lobe structure without the zonal anatomy typically in human prostate (11). On T2weighted images, the gland tissue was of heterogeneous hyperintense signal intensity (SI) in comparison with SI of the obturator internus muscles. Some glandular tissue showed striated hyperintense SI in a radial pattern from the urethra to the capsule. On T1-weighted images, the prostate gland showed homogeneous and isointense SI. On contrast-enhanced T1-weighted images, the parenchyma appeared homogenous and mildly hyperintense SI as well as isointense striated radial structure corresponding to the same structure of hyperintense SI on T2-weighted images. Around the prostatic urethra there was focal area with SI of high enhancement in all dogs, indicating rich of microvasculature along the urethral wall.

\section{Early MRI findings of prostate infarction}

Prostate infarcts were detected at 1 week after PAE in all ten dogs, and at 2 weeks and 1 month in nine dogs. In the only one $\mathrm{dog}$, in which the prostate infarct was not detectable after 1 week, the prostate showed continued decrease in size at 2-week and 1-month follow-ups. On contrast-enhanced T1-weighted images, infarcts exhibited as multiple, round, oval, or irregular non-enhanced lesions surrounded by rims of high SI, representing perinecrotic areas of inflammation, such as hyperemia and edema, and atrophy (12). At 1-week follow-ups, all infarcts showed smooth and sharply demarcated margin (Figure 1). Subsequently, the size of infarcts gradually decreased, accompanying with shrinkage of the prostate gland (Figure 2). The typical findings of MRI SI and the morphological changes are displayed in Figure 1 and Figure 2.

On T1-weighted images, lesions involved a mixture of SI with annular or irregular focal areas of faint hyperintense signal indicative of intraprostatic hemorrhage surrounding or within ischemic necrosis identified with isointense signal. The faint hyperintense areas tented to become smaller and isointense with time. When cavity formation occurred at 1 month after PAE in the initially large infarcts, the local signal exhibited isointense or hypointense on T1-weighted images (Figure 1A,D, G, f).

On T2-weighted images, all infarcts were identified with hyperintense signal with well-defined margin at 1 week after PAE. Since the inflammation rims showed on 

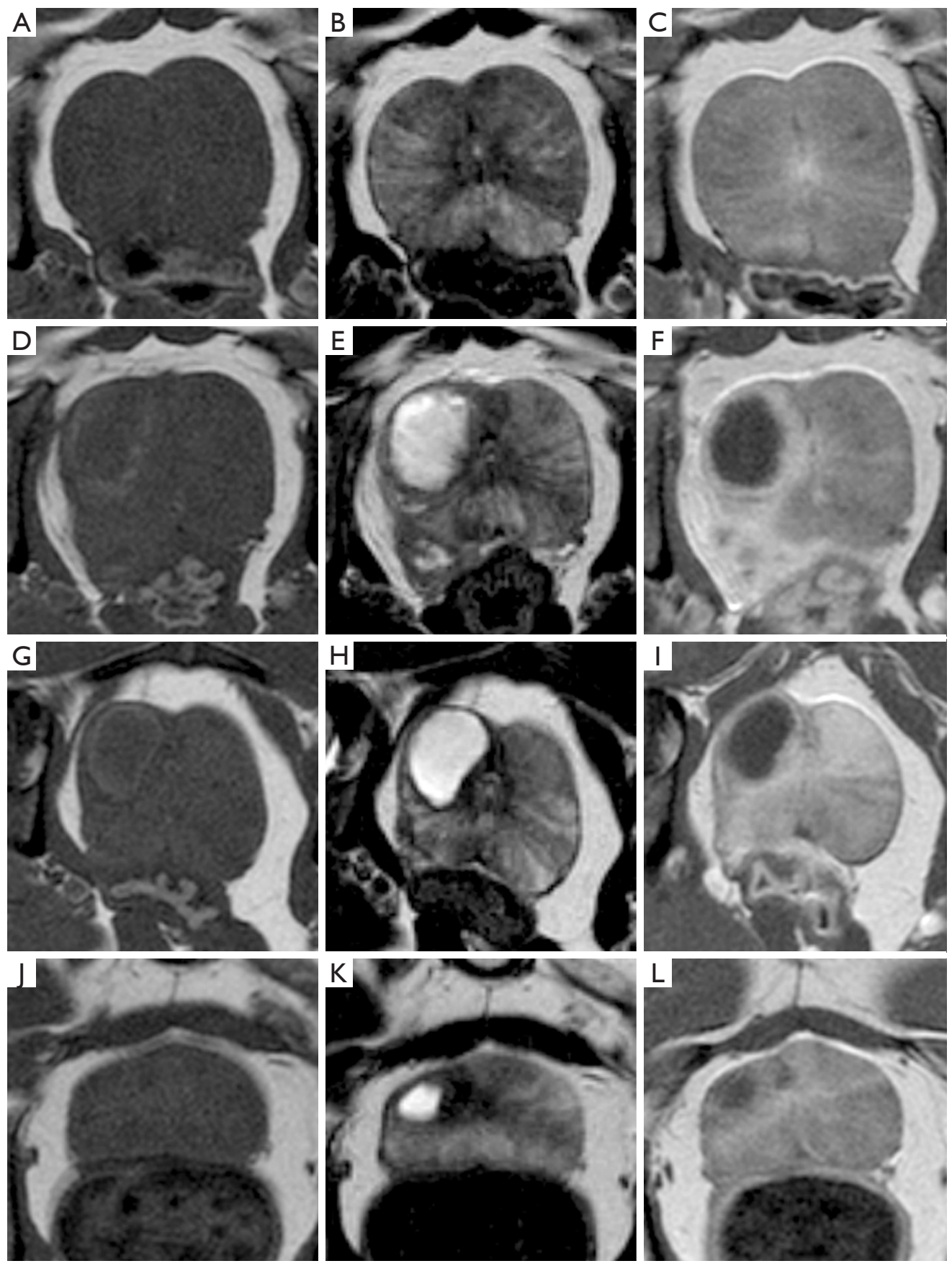

Figure 1 Typical findings of pathological responses to PAE in MRI. MRI includes baseline data (A,B,C) and 1 week (D,E,F), 2 weeks (G,H,I) and 1 month (J,K,L) after PAE, with T1-weighted (left column), T2-weighted (middle column) and contrast-enhanced T1-weighted (right column) images. T1-weighted images show annular area of hemorrhage in faint hyperintense SI surrounding the central area of infarction in isointense SI (D,G). T2-weighted images show infarcts in hyperintense SI with well-defined margin (E,H). Contrast-enhanced T1-weighted images clearly delineate in the infarcts with rims of high SI, indicative of perinecrotic areas of inflammation (F,I), which disappeared at 1 month after PAE (L). Note that the lesions on T2-weighted images appear larger in size than the true infarction lesions shown in contrastenhanced T1-weighted images. At 1-month follow-up, small cavity formation shows isointense SI on T1-weighted image (J) and highly increased signal with well-defined margin on T2-weighted image (K). PAE, prostatic artery embolization; MRI, magnetic resonance imaging; SI, signal intensity. 

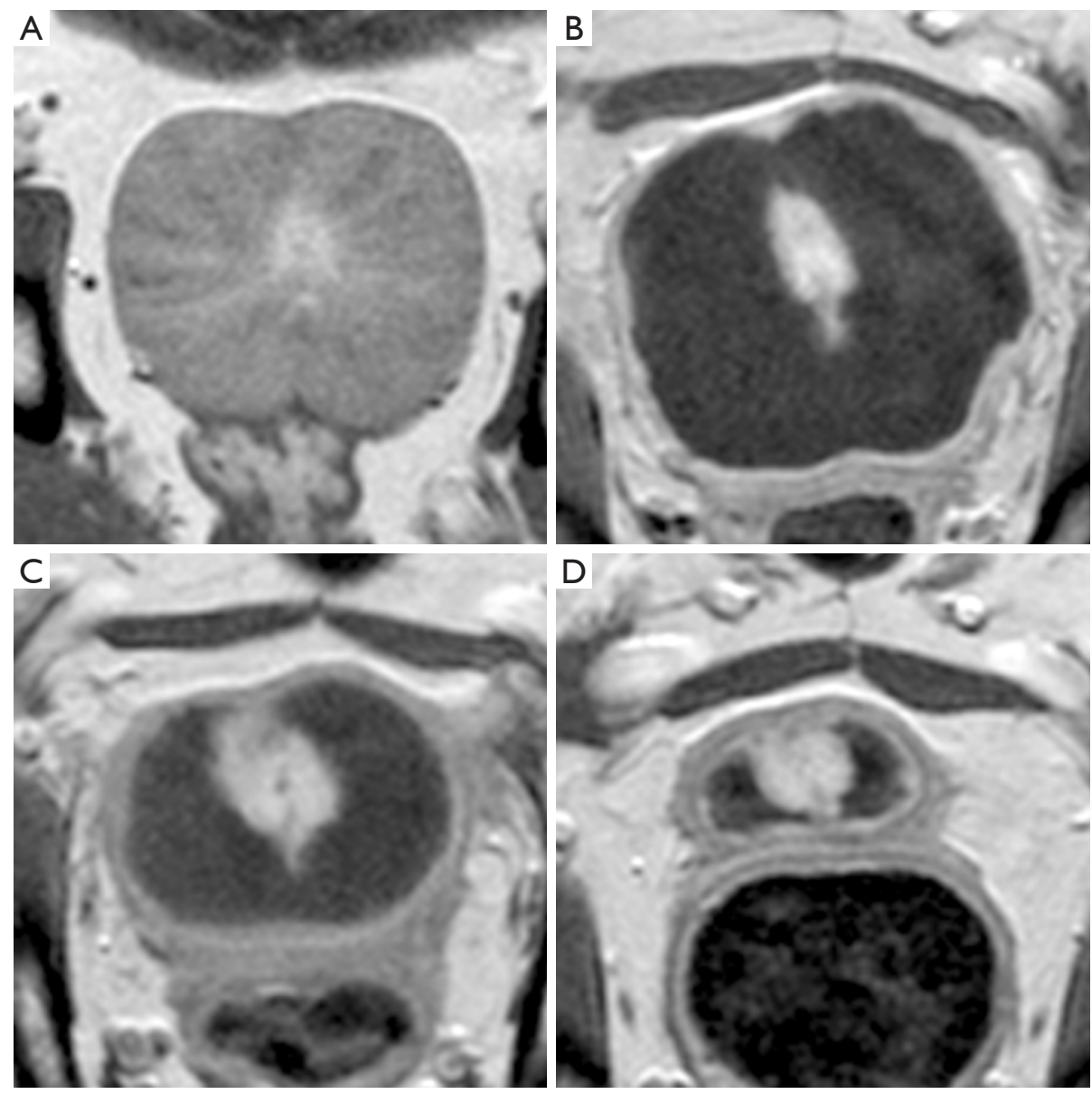

Figure 2 Changes of prostate infarction with time. (A) Baseline image; (B) 1 week; (C) 2 weeks; (D) 1 month post-PAE. Note that a masslike infarct with smooth and sharply demarcated margin gradually decreases in size with time while the prostate keeps shrinking and a hyperintense SI rim remains at 1 month after PAE (D). PAE, prostatic artery embolization; SI, signal intensity.

contrast-enhanced T1-weighted images partially exhibited hyperintense signal on T2-weighted images, the size of the lesion on T2-weighted images appeared slightly larger when comparing with contrast-enhanced T1-weighted images. The hyperintense SI tended to decrease with time and finally became hypointense signal (Figure $1 B, E, H, K$ ). When cavity formed at 1 month after PAE, the hyperintense SI became highly increased with well-defined margin.

\section{PV reduction and SUMIA}

Compared with the $\mathrm{PV}$ at baseline, significant $\mathrm{PV}$ reduction was found at both 2 weeks and 1 month after $\mathrm{PAE}(\mathrm{P}<0.001)$ (Table 1). Furthermore, the prostate continued to shrink substantially between 2 weeks and 1 month after PAE $(\mathrm{P}=0.004)$. However, no significant change in $\mathrm{PV}$ was observed at 1 week post-PAE in comparison with baseline data. SUMIA was obtained by calculation of each area of infarct of all prostate sections on contrast-enhanced T1weighted images (Figure 3). The mean SUMIA significantly decreased $(\mathrm{P}=0.002)$ from 1 to 2 weeks and continued to decrease substantially $(\mathrm{P}=0.039)$ from 2 weeks to 1 month after PAE (Table 2). Similarly, significant decrease in the mean prostate infarction percentage $(\mathrm{P}=0.002)$ was observed at both 2 weeks and 1 month when compared to the data at 1 week. Spearman's rank correlation test demonstrated a strong correlation of PV reduction rate at 1 month after PAE with the infarction percentage at 1 week $(r=0.880$, $\mathrm{P}=0.001)$ and a moderately strong correlation at 2 weeks $(\mathrm{r}=0.733, \mathrm{P}=0.016)$. Linear regression analysis showed coefficient of determination $\left(\mathrm{r}^{2}\right)$ of $0.775(\mathrm{P}=0.001)$ at 1 week and $0.538(\mathrm{P}=0.016)$ at 2 weeks (Table 3$)$. In addition, 
Table 1 Summary of MRI measurements (means \pm standard deviation) and changes in major parameters

\begin{tabular}{lcccc}
\hline Variable & Baseline & 1 week & 2 weeks & 1 month \\
\hline Prostate volume $(\mathrm{mL})$ & $19.69 \pm 2.75$ & $19.22 \pm 4.77$ & $12.23 \pm 2.81^{*}$ & $8.75 \pm 2.57^{\star}$ \\
Prostate volume reduction $(\%)$ & - & $2.54 \pm 20.05$ & $38.49 \pm 7.78^{\star}$ & $55.42 \pm 11.23^{\star}$ \\
SUMIA $\left(\mathrm{cm}^{2}\right)$ & - & $14.45 \pm 16.85$ & $3.75 \pm 6.31^{*}$ & $0.68 \pm 0.87^{\star}$ \\
SUMPA $\left(\mathrm{cm}^{2}\right)$ & - & $66.44 \pm 18.69$ & $45.21 \pm 8.98^{\star}$ & $36.75 \pm 9.29^{*}$ \\
Infarction percentage $(\%)$ & - & $18.16 \pm 16.95$ & $7.79 \pm 11.30^{*}$ & $2.30 \pm 3.42^{*}$ \\
\hline
\end{tabular}

*, significant difference $(P$ value $<0.05)$. MRI, magnetic resonance imaging; SUMIA, sum of total infarct area in all sections of the prostate; SUMPA, sum of prostate areas in all sections.
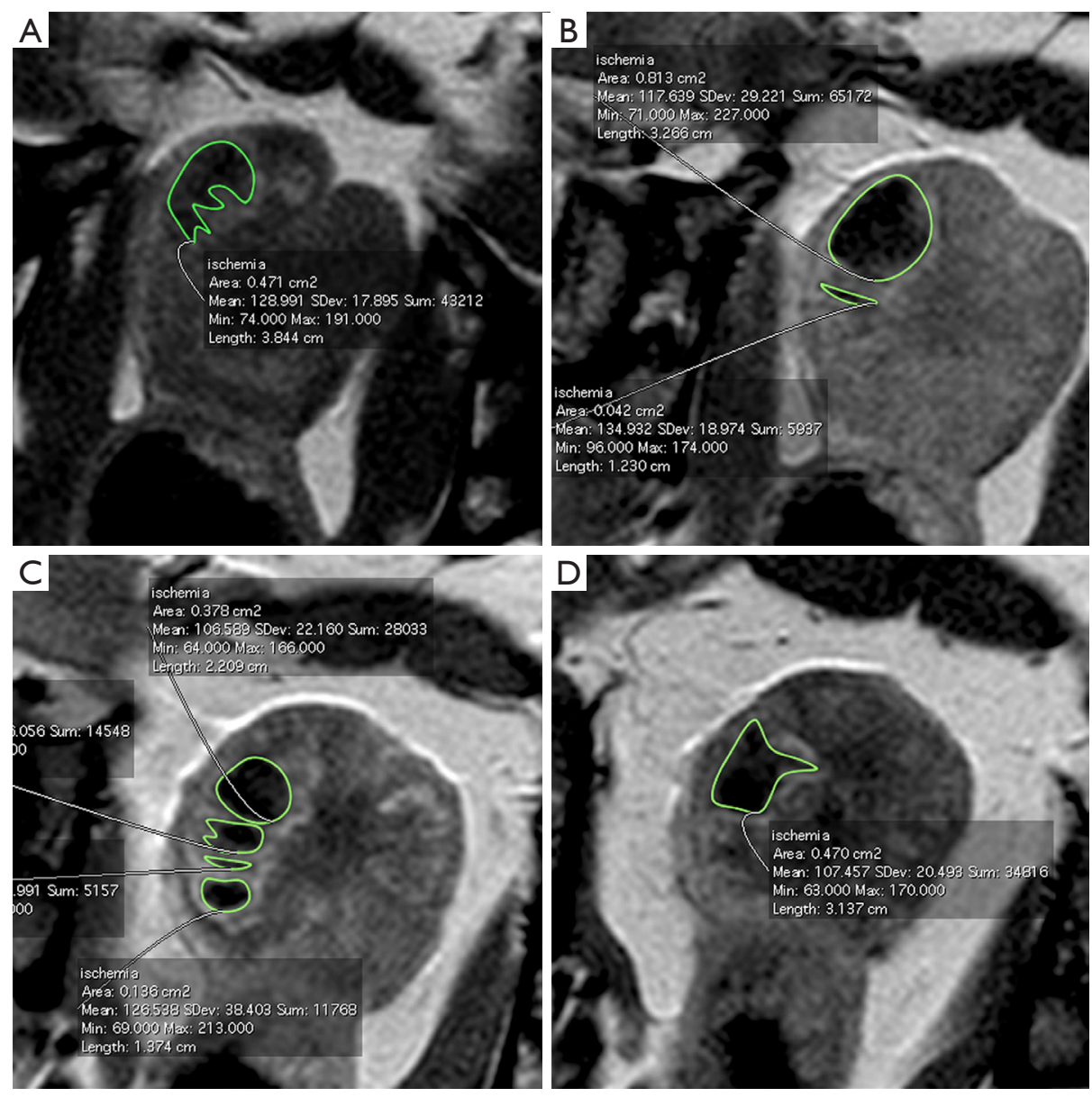

Figure 3 Measurement of SUMIA with OsiriX software. Contrast-enhanced T1-weighted images of 4 contiguous sections at 1-week follow-up (A,B,C,D) show the infarction lesions of complex form in morphology, which consist of multiple separated "sister lesions" (B,C). The infarction areas are outlined to generate various regions of interest using OsiriX software for calculation of SUMIA. SUMIA, sum of infarct areas.

Spearman's rank correlation test indicted that $\mathrm{PV}$ reduction rate had a significant correlation with decrease in SUMIA $(\mathrm{r}=0.854, \mathrm{P}=0.002)$ at 1 month after $\mathrm{PAE}$, with a coefficient of determination of $\mathrm{r}^{2}=0.729(\mathrm{P}=0.002)$.

\section{Discussion}

The technique of contrast enhanced MRI in the present study involves an intravenous bolus injection of a contrast medium immediately followed by image acquisition using 
Table 2 Comparison of MRI measurements to baseline data at different time points

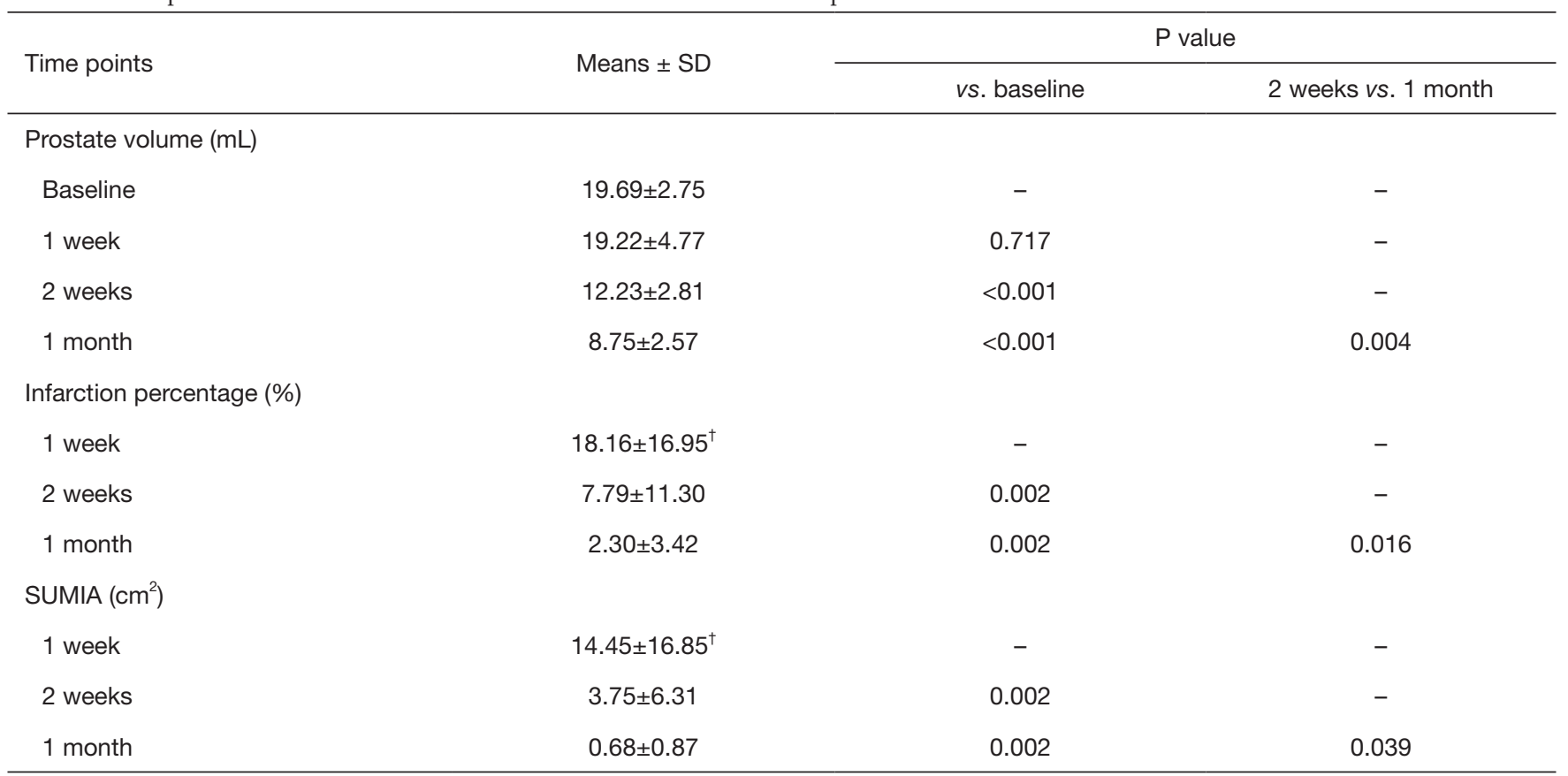

${ }^{\dagger}$, baseline data in infarction percentage and SUMIA correspond to 1 week; MRI, magnetic resonance imaging; SD, standard deviation; SUMIA, sum of total infarct area in all sections of the prostate.

Table 3 Correlation of PV reduction at 1 month after PAE with SUMIA and prostate infarction percentage

\begin{tabular}{|c|c|c|c|c|}
\hline Predictor variable & Correlation coefficient $(r)$ & $P$ value & Coefficient of determination $\left(r^{2}\right)$ & $P$ value \\
\hline 1 week & 0.880 & 0.001 & 0.775 & 0.001 \\
\hline 2 weeks & 0.733 & 0.016 & 0.538 & 0.016 \\
\hline \multicolumn{5}{|l|}{ SUMIA $\left(\mathrm{cm}^{2}\right)$} \\
\hline 2 weeks & 0.735 & 0.015 & 0.540 & 0.015 \\
\hline
\end{tabular}

PV, prostate volume; PAE, prostatic artery embolization; SUMIA, sum of total infarct area in all sections of the prostate.

T1-weighted turbo spin echo sequence. After perfusion of contrast medium into the prostate capillaries, the low molecular weight agent quickly diffuses into extracellular space, reducing the relaxation time of water protons and displaying a local enhancement with slightly high signal on T1 images. In the ischemic necrotic tissue, perfusion of the contrast agent fails due to microvascular obstruction, resulting in the lack of local enhancement. By contrast, inflammation reaction surrounding the affected tissue, such as hyperemia and edema, increases the capillary permeability and enlarges the extravascular space. As a result, excessive contrast medium diffusing in the local area leads to hyperenhancement compared to the unaffected tissue. Contrast-enhanced MRI has been validated as a standard technique to detect and assess prostate infarct not only in clinical practice $(5,6,13)$ but also in canine studies, in which prostate infarction detected in contrastenhanced MRI had been demonstrated with a strong correlation with pathological findings (12). In the present study, it clearly delineated the acute infarcts of various sizes with well-defined margin and demonstrated the changes in morphology with time, which was consistent with the findings in the previous animal study (12). Although T2-weighted images are also sensitive to acute prostate 
infarction displaying homogenous high signal in early phase, they usually overestimate the size of the infarcts because the region of high signal includes not only ischemic necrosis but the surrounding area of inflammation reaction as well. T1-weighted images, however, provide limited information, mainly showing the focal area of high signal indicative of hemorrhage surrounding and/or within the early ischemic necrosis due to vascular endothelial damage and accumulation of extravasated erythrocytes in the extracellular space. Similar findings were also observed in both dogs and human patients $(12,14)$.

Accurate measurement of infarct volume using contrastenhanced T1 imaging is technically challenging, particularly in early detection after PAE. Although some well-designed software, such as OsiriX and DynaCAD Prostate ${ }^{\circledR}$, have been reported in human patients $(5,15,16)$, they are commonly used in segmentation of relative large 3-dimensional lesions, which extend to at least two contiguous axial sections. Thus, it is impossible to determine the size of the focal or flat infarcts distributing in a single section. In addition, in lesions of complex form in morphology (Figure 3), which consist of multiple separated "sister lesions" merging at some points, it is difficult to measure the infarct volume accurately with the aforementioned software using either automated or manual segmentation methods $(15,17)$. Herein the authors described SUMIA as a simple alternative measure to document the infarct size instead of prostate infarction volume, which has a higher accuracy and potential in future clinical practice.

In pathology, infarction is defined as tissue necrosis due to prolonged ischemia and involves a dynamic process divided into 4 phases: infarction evolving, acute infarction, healing infarction, and healed infarction (18). Coagulative necrosis is a typical pathological finding of infarction, which usually takes at least 6 hours for microscopic identification, depending on the presence of collateral circulation into the ischemic region, persistent or intermittent vascular occlusion and tissue sensitivity of the tissue (19). Although studies on myocardial infarction have demonstrated pathological changes at different time points: acute ( 6 h-7 days), healing (7-28 days), and healed (29 days or more) (18); the pathological features with regard to prostate infarction is unknown. Accordingly, the authors used 1 week after PAE as the first time point in early detection of prostate infarction and followed up its morphological changes through 1 month. The findings that the mean SUMIA significantly decreased from 1 to 2 weeks after PAE suggested that healing of the prostate infarction occurred during this period. Furthermore, the authors observed that $\mathrm{PV}$ reduction rate at 1 month after PAE had a significant correlation with decrease in SUMIA $(\mathrm{r}=0.854, \mathrm{P}=0.002)$, suggesting that the dissolving infarction characterized by decrease in SUMIA is a main mechanism behind the shrinkage of prostate after PAE. Given that there was no obvious change in the mean PV between baseline and 1 week after PAE, significant reduction in infarction size during the same period would not be likely. This observation supported the speculation that 1 week after PAE was a cut-off time, at which complete prostate infarction ended and the phase of healing infarction started. This was in agreement with pathological features of myocardial infarction as mentioned above. Additionally, it has been demonstrated that prostate infarction volume detected at 1 week after treatment by means of contrastenhanced T1 imaging was consistent with size of infarction in pathological examination (12). In this study, canine prostate ischemic necrosis was induced by vascular-targeted photodynamic therapy and the volume of ischemic necrosis at 1 week after treatment was identified to have a strong correlation volume with pathological infarct size $(\mathrm{r}=0.87$, $r^{2}=0.75$, with a linear slope of 0.99 ) (12). Therefore, 1 week after PAE would be an ideal and reliable time point in early detection of prostate infarction. However, it should be emphasized that there exists much difference regarding tissue tolerance to ischemia between myocardium and prostate gland, especially in term of the terminal blood supply pattern in myocardium and the anatomy of the potential collateral circulation of the prostate. Further studies are needed to address the tolerance of the prostate to ischemia in human patients.

The findings in the present study indicated that prostate infarction percentage at 1 or 2 weeks after PAE may predict the therapeutic effect (shrinkage of the prostate) after treatment due to its strong correlation with PV reduction at 1 month (Table 3). However, the predictability of prostate infarction percentage at 1 week seemed to be superior to that at 2 weeks after PAE due to their differences of the correlation coefficient and coefficient of determination observed in the present study. Although SUMIA at 1 or 2 weeks in the present study showed the predictability quite similar to the prostate infarction percentage at the same time point (Table 3), prostate infarction percentage that was defined as the ratio of SUMIA/SUMPA in this study is more useful in clinical practice because human patients undergoing PAE usually have a wide range of prostate size. By the time of this writing, only one clinical trial by Wang and colleagues (20) has demonstrated the predictive role 
of prostate infarct size at 1 week after PAE. Most studies have been evaluating the prostate infarction at 2-4 weeks through 3 months after PAE. Although the prostate infarction percentage has been identified to have association with higher clinical success in removal of the indwelling catheter (5) and with lower International Prostate Symptom Score in subsequent follow-ups (4), controversy remains due to different patient selection criteria and distinct study design and statistical analysis $(6,15)$. More recently, detection of prostate ischemia at 24 hours after PAE has gained interest in clinical prediction by means of contrastenhanced ultrasonography or pharmacokinetic analysis with dynamic contrast-enhanced MRI $(8,9)$; however, the clinical predictive role failed to be established even if a close relationship of the prostate infarction percentage at 24 hours post-PAE and subsequent prostate shrinkage (8). Obviously, further studies are needed to address the best time point in detection of prostate infarction and its prediction role in clinical practice. It should be emphasized that although all dogs used in the present study were with spontaneous BPH, the canine model has its inherent limitations due to the lack of LUTS and BOO as like in human patients (10). Thus, shrinkage of the prostate was the only outcome parameter in evaluation of therapeutic effects in the present study.

This study had several other limitations. Total number of ten dogs was a relatively small sample size, partially due to the limited commercial availability of the older male beagles. The follow-up period was short, so that observation of changes in signals on MRI sequences after 1 month following PAE was not available. Due to the limited number of animals, we were not able to conduct pathological evaluation at acute infarction phase, e.g., 1 week after PAE, in comparison with the MRI findings. Although different spherical embolic agents with similar size were used in $\mathrm{PAE}$, which might result in various degrees of inflammation and infarction; the present study focused more on the morphological changes of infarction after PAE than the real size of infarction induced with embolic agents.

\section{Conclusions}

Prostate infarction is one of major early pathological responses to PAE in canine BPH model. SUMIA can be used as surrogate parameter to measure prostatic ischemia due to its more accuracy in irregular and multiples infarcts. One week after PAE is an ideal time point for MRI evaluation of acute prostate infarction. Prostate infarction at 1 week can be used as an independent variable in prediction of prostate shrinkage at 1 month after PAE. More pathological studies in animal experiments and clinical trials are needed to further address the predictive role of prostate infarction in PAE clinical outcomes.

\section{Acknowledgments}

Acknowledge to the surgical and anesthesia technical staff from Jesús Usón Minimally Invasive Surgery Centre for its commitment in the study.

Funding: This study was supported by MicroVention, Inc., A TERUMO Group Company and in part by grant IB18129 from Plan Regional de Investigación, Consejería de Economía, Ciencia y Agenda Digital, Junta de Extremadura and Fondo Europeo de Desarrollo Regional.

\section{Footnote}

Reporting Checklist: The authors have completed the ARRIVE reporting checklist. Available at http://dx.doi. org/10.21037/tau-20-1320

Data Sharing Statement: Available at http://dx.doi. org/10.21037/tau-20-1320

Conflicts of Interest: All authors have completed the ICMJE uniform disclosure form (available at http://dx.doi. org/10.21037/tau-20-1320). The authors have no conflicts of interest to declare.

Ethical Statement: The authors are accountable for all aspects of the work in ensuring that questions related to the accuracy or integrity of any part of the work are appropriately investigated and resolved. Experiments were performed under a project license (No. 2018209010001587 and No. 2018209010008108) granted by Regional Ethic Committee of Junta de Extremadura, in compliance with Spanish national guidelines for the care and use of animals.

Open Access Statement: This is an Open Access article distributed in accordance with the Creative Commons Attribution-NonCommercial-NoDerivs 4.0 International License (CC BY-NC-ND 4.0), which permits the noncommercial replication and distribution of the article with the strict proviso that no changes or edits are made and the original work is properly cited (including links to both the formal publication through the relevant DOI and the license). See: https://creativecommons.org/licenses/by-nc-nd/4.0/. 


\section{References}

1. Wang M, Guo L, Duan F, et al. Prostatic arterial embolization for the treatment of lower urinary tract symptoms caused by benign prostatic hyperplasia: A comparative study of medium- and large-volume prostates. BJU Int 2016;117:155-64.

2. Sun F, Crisóstomo V, Báez-Díaz C, et al. Prostatic Artery Embolization (PAE) for Symptomatic Benign Prostatic Hyperplasia (BPH): Part 2, Insights into the Technical Rationale. Cardiovasc Intervent Radiol 2016;39:161-9.

3. Sun F, Lucas-Cava V, Sánchez FM. Clinical Predictive Factors in Prostatic Artery Embolization for Symptomatic Benign Prostatic Hyperplasia: a Comprehensive Review. Transl Androl Urol 2020;9:1754-68.

4. Bilhim T, Pisco J, Pereira JA, et al. Predictors of clinical outcome after prostate artery embolization with spherical and nonspherical polyvinyl alcohol particles in patients with benign prostatic hyperplasia. Radiology 2016;281:289-300.

5. Kisilevzky N, Faintuch S. MRI assessment of prostatic ischaemia: best predictor of clinical success after prostatic artery embolisation for benign prostatic hyperplasia. Clin Radiol 2016;71:876-82.

6. Frenk NE, Baroni RH, Carnevale FC, et al. MRI findings after prostatic artery embolization for treatment of benign hyperplasia. AJR Am J Roentgenol 2014;203:813-21.

7. Brook OR, Faintuch S, Brook A, et al. Embolization therapy for benign prostatic hyperplasia: Influence of embolization particle size on gland perfusion. J Magn Reson Imaging 2013;38:380-7.

8. Moschouris H, Stamatiou K, Malagari K, et al. The value of contrast-enhanced ultrasonography in detection of prostatic infarction after prostatic artery embolization for the treatment of symptomatic benign prostatic hyperplasia. Diagn Interv Radiol 2019;25:134-43.

9. Franiel T, Aschenbach R, Trupp S, et al. Prostatic Artery Embolization with 250- $\mu \mathrm{m}$ Spherical PolyzeneCoated Hydrogel Microspheres for Lower Urinary Tract Symptoms with Follow-up MR Imaging. J Vasc Interv Radiol 2018;29:1127-37.

10. Sun F, Báez-Díaz C, Sánchez-Margallo FM. Canine prostate models in preclinical studies of minimally invasive interventions: Part II, benign prostatic hyperplasia models. Transl Androl Urol 2017;6:547-55.

11. Sun F, Báez-Díaz C, Sánchez-Margallo FM. Canine prostate models in preclinical studies of minimally invasive interventions: Part I, canine prostate anatomy and prostate cancer models. Transl Androl Urol 2017;6:538-46.

12. Huang Z, Haider MA, Kraft S, et al. Magnetic resonance imaging correlated with the histopathological effect of $\mathrm{Pd}$ bacteriopheophorbide (Tookad) photodynamic therapy on the normal canine prostate gland. Lasers Surg Med 2006;38:672-81.

13. Haider MA, Davidson SRH, Kale A V, et al. Prostate gland: MR imaging appearance after vascular targeted photodynamic therapy with palladiumbacteriopheophorbide. Radiology 2007;244:196-204.

14. Zhang H, Shen Y, Pan J, et al. MRI features after prostatic artery embolization for the treatment of mediumand large-volume benign hyperplasia. Radiol Med 2018;123:727-34.

15. Lin YT, Amouyal G, Correas JM, et al. Can prostatic arterial embolisation (PAE) reduce the volume of the peripheral zone? MRI evaluation of zonal anatomy and infarction after PAE. Eur Radiol 2016;26:3466-73.

16. Ali R, Gabr A, Mouli SK, et al. MR imaging findings of the prostate gland following prostate artery embolization: results from a prospective phase 2 study. Abdom Radiol (NY) 2019;44:713-22.

17. Turkbey B, Fotin SV, Huang RJ, et al. Fully automated prostate segmentation on MRI: comparison with manual segmentation methods and specimen volumes. AJR Am J Roentgenol 2013;201:W720-9.

18. Thygesen K, Alpert JS, White HD. Universal definition of myocardial infarction. Eur Heart J 2007;28:2525-38.

19. Alpert JS, Thygesen K, Antman E, et al. Myocardial infarction redefined--a consensus document of The Joint European Society of Cardiology/American College of Cardiology Committee for the redefinition of myocardial infarction. J Am Coll Cardiol 2000;36:959-69. Erratum in: J Am Coll Cardiol 2001 Mar 1;37(3):973.

20. Wang MQ, Zhang JL, Xin HN, et al. Comparison of Clinical Outcomes of Prostatic Artery Embolization with 50- $\mu \mathrm{m}$ Plus 100- $\mu \mathrm{m}$ Polyvinyl Alcohol (PVA) Particles versus 100- $\mathrm{mm}$ PVA Particles Alone: A Prospective Randomized Trial. J Vasc Interv Radiol 2018;29:1694-702.

Cite this article as: Lucas-Cava V, Sánchez-Margallo FM, García-Martínez V, López-Sánchez C, Báez-Díaz C, DávilaGómez L, Lima-Rodríguez JR, Sun F. Prostatic artery embolization: magnetic resonance image (MRI) findings in the early detection of prostate infarction in a canine spontaneous benign prostatic hyperplasia model. Transl Androl Urol 2021;10(2):869-878. doi: 10.21037/tau-20-1320 